\title{
SOME NOTES ON TWO TYPES OF MINIMAL REPAIR
}

\author{
M. S. FINKELSTEIN, ${ }^{*}$ Electropribor Institute, St Petersburg
}

\begin{abstract}
Two types of minimal repair are discussed. After statistical minimal repair, the state of a system is statistically identical to what it was just before the failure. Physical minimal repair restores the failed unit to its exact physical condition before the failure. Several examples are given.
\end{abstract}

PHYSICAL MINIMAL REPAIR; STATISTICAL MINIMAL REPAIR; MAINTENANCE

AMS 1991 SUBJECT CLASSIFICATION: PRIMARY 60K10

\section{Introduction}

Consider a system with an absolutely continuous failure-time distribution function $\mathscr{F}(x)$ and failure rate function $\lambda(x)$. A concept of minimal repair was introduced by Barlow and Proschan [2]: if a failure occurs at time $t$ then, after the minimal repair, the survival probability to time $t+s$ is $(1-\mathscr{F}(t+s) /(1-\mathscr{F}(t))$ and the failure rate function is $\lambda(t+s)$, $s \geqq 0$. This means that after the repair the state of a system is statistically identical to the state just before the failure. That is why this type of repair was called by Bergman [3] statistical minimal repair (SMR), differing from physical minimal repair (PMR) when the failed unit is restored to the exact physical condition it was in just before the failure. Later Arjas and Norros [1] introduced the so-called F-minimal repair (FMR), where F stands for the information that identifies the state of a failed device. FMR is a more general notion: when we have no information to hand it is SMR, when we have complete information, idealizing the situation of course, it is PMR. Several particular cases of FMR were treated by Finkelstein [4].

It should be noted that there are two interpretations of SMR. The first one was introduced to mimic the behaviour of a large system when one of its many components is replaced or repaired. The second one (which has not so far received proper attention) describes the situation when the whole system after the failure is replaced by an identical one that had functioned for the same time and in the 'same way' but had not failed (loaded redundancy). This second interpretation will help us to treat the examples below.

Natvig [5] conjectured that for the two types of minimal repair the following inequality holds:

$$
\overline{\mathscr{F}}_{\mathrm{SMR}}(x) \geqq \overline{\mathscr{F}}_{\mathrm{FMR}}(x),
$$

where $\mathscr{F}_{\mathrm{SMR}}(x)$ and $\mathscr{F}_{\mathrm{FMR}}(x)$ are the distribution functions of the remaining system lifetimes just after SMR and FMR respectively, $\mathscr{F}(x) \equiv 1-\mathscr{F}(x)$.

The inequality (1) (not yet proved) means that the remaining system lifetime after SMR is stochastically longer than after FMR. Some examples dealing with (1) are presented below.

Received 6 August 1991; revision received 21 October 1991.

* Central Research Institute 'Electropribor', Malaya Posadskaya str. 30, 197046 St Petersburg, Russia. Postal address: Socialisticheskaya str. 6, 17, 191002 St Petersburg, Russia. 


\section{Three examples}

A. Single unloaded redundancy. Let the distribution function of an item be $\mathscr{F}_{i}(x)=$ $1-e^{-\lambda x}$. Then for the system of two identical elements, $\mathscr{F}_{s}(x)=1-e^{-\lambda x}(1+\lambda x)$. It is easily seen that the failure rate function corresponding to the remaining lifetime is $\lambda$ for FMR (the information to hand leads to the replacement of only one element after the failure of the system) and $\lambda s(t)=\lambda^{2} t /(1+\lambda t)$ for SMR. Finally, because $\lambda^{2} t /(1+\lambda t)<\lambda$ for all $t$, one can derive (1).

B. Single loaded redundancy. Let the distribution function of the first element be $\mathscr{F}_{1}(x)=1-e^{-\lambda_{1} x}$ and of the second $\mathscr{F}_{2}(x)=2-e^{-\lambda_{2} x}$. Assume the system has failed at time $t$. Then the following theorem holds.

Theorem. For each $t \geqq 0$ the following inequality holds:

$$
\overline{\mathscr{F}}_{\text {SMR }}(x) \geqq \overline{\mathscr{F}}_{\text {FMR }}(x) \text {, }
$$

where $\mathscr{F}_{\mathrm{SMR}}(x)$ and $\mathscr{F}_{\mathrm{FMR}}^{t}(x)$ are the distribution functions of the remaining system lifetimes just after SMR and FMR respectively at time $t$.

Proof. Let $\lambda_{1}>\lambda_{2}$. In accordance with the definition of SMR and the second interpretation of minimal repair mentioned above, we can derive the probabilities of a system being just after SMR in respectively the states where the first element is operating (the second has failed), $P_{1}^{\text {SMR }}(t)$; the second element is operating (the first has failed), $P_{2}^{\text {SMR }}(t)$; and both elements are operating, $P_{1,2}^{\text {SMR }}(t)$. We have

$$
\begin{aligned}
& P_{2}^{\mathrm{SMR}}(t)=\frac{e^{-\lambda_{1} t}\left(1-e^{-\lambda_{2} t}\right)}{e^{-\lambda_{1} t}+e^{-\lambda_{2} t}-e^{-\left(\lambda_{1}+\lambda_{2}\right) t}}, \\
& P_{2}^{\mathrm{SMR}}(t)=\frac{e^{-\lambda_{2} t}\left(1-e^{-\lambda_{1} t}\right)}{e^{-\lambda_{1} t}+e^{-\lambda_{2} t}-e^{-\left(\lambda_{1}+\lambda_{2}\right) t},} \\
& P_{1,2}^{\mathrm{SMR}}(t)=\frac{e^{-\left(\lambda_{1}+\lambda_{2}\right) t}}{e^{-\lambda_{1} t}+e^{-\lambda_{2} t}-e^{-\left(\lambda_{1}+\lambda_{2}\right) t}}
\end{aligned}
$$

After FMR, by definition, the system can obviously be in only one of two states, with probabilities $P_{1}^{\mathrm{FMR}}(t)$ and $P_{2}^{\mathrm{FMR}}(t)$. It is easily seen that

$$
\begin{aligned}
& P_{1}^{\mathrm{FMR}}(t)=\frac{\lambda_{1} e^{-\lambda_{1} t}\left(1-e^{-\lambda_{2} t}\right)}{\lambda_{1} e^{-\lambda_{1} t}\left(1-e^{-\lambda_{2} t}\right)+\lambda_{2} e^{-\lambda_{2} t}\left(1-e^{-\lambda_{1} t}\right)}, \\
& P_{2}^{\mathrm{FMR}}(t)=\frac{\lambda_{2} e^{-\lambda_{2} t}\left(1-e^{-\lambda_{1} t}\right)}{\lambda_{2} e^{-\lambda_{2} t}\left(1-e^{-\lambda_{1} t}\right)+\lambda_{1} e^{-\lambda_{1} t}\left(1-e^{-\lambda_{2} t}\right)} .
\end{aligned}
$$

Remembering that $\lambda_{1}>\lambda_{2}$, we obtain

$$
P_{1}^{\mathrm{FMR}}(t)>\frac{e^{-\lambda_{1} t}\left(1-e^{-\lambda_{2} t}\right)}{e^{-\lambda_{1} t}+e^{-\lambda_{2} t}-e^{-\left(\lambda_{1}+\lambda_{2}\right) t}}=P_{1}^{\mathrm{SMR}}(t) .
$$

The inequality means that FMR with greater probability than SMR brings the system to the state where the worst element is functioning. Combining (8) with

$$
\begin{gathered}
P_{1}^{\mathrm{FMR}}(t)+P_{2}^{\mathrm{FMR}}(t)=1 \\
P_{1}^{\mathrm{SMR}}(t)+P_{2}^{\mathrm{SMR}}(t)+P_{1,2}^{\mathrm{SMR}}(t)=1
\end{gathered}
$$

completes the proof of the theorem.

Corollary. The inequality (2) for the systems investigated leads to the inequality (1).

Because $t$ is arbitrary the proof is obvious. 


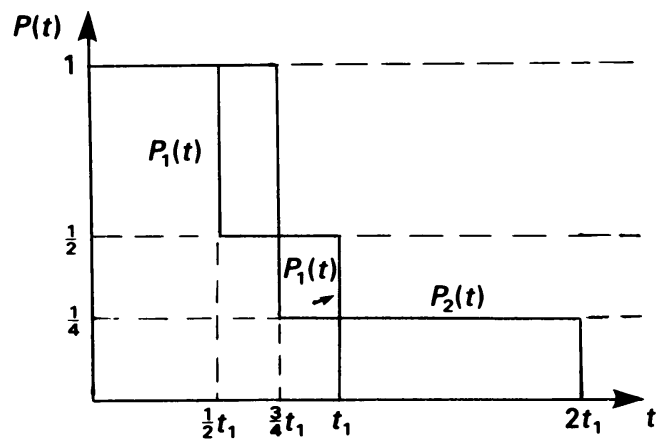

Figure 1

The result can be easily generalized for $n$-tuple redundancy $(n=2,3, \cdots)$.

C. Single loaded redundancy with arbitrary $\mathscr{F}_{1}(x)$ and $\mathscr{F}_{2}(x)$. One might imagine that (2) holds for all redundant systems, but a counterexample shows that this is not so. Let $P_{1}(t) \equiv 1-\mathscr{F}_{1}(t), P_{2}(t) \equiv 1-\mathscr{F}_{2}(t)$ be as in Figure 1 (we do not need for this counterexample the assumption of absolute continuity of the distribution functions, which was made only for simplicity).

Suppose that the system had failed at $t=\frac{3}{4} t_{1}$. If follows from the figure that with probability 1 it is the second element that has failed. After FMR the system will function for $2 t_{1}-\frac{3}{4} t_{1}=1 \frac{1}{4} t_{1}$. It is obvious that after SMR it will function stochastically for a shorter period of time because only some of the realizations will have length $1 \frac{1}{4} t_{1}$; others will be $\frac{1}{4} t_{1}$. Thus the inequality (2) does not hold for $t=\frac{3}{4} t_{1}$, although it is easily seen that it holds for $t=t_{1}$.

\section{Conclusion}

Both concepts of minimal repair can be helpful for probability analyses of repairable systems. SMR is good for describing the replacement of the failed system by an identical one. FMR is preferable for the replacement of the failed units of a system.

The inequality (2) holds for two-unit systems because $1-e^{-\lambda_{1} x}>1-e^{-\lambda_{2} x}$, for all $x>0$. Presumably this conclusion is the same for arbitrary $\mathscr{F}_{1}(x), \mathscr{F}_{2}(x)$, if $\bar{F}_{2}(x) \geqq \bar{F}_{1}(x)$, for all $x \geqq 0$. If this inequality does not hold for all $x>0$, then as shown by the counterexample there can be $x$ and $t$ that change the sign of inequality (2).

\section{References}

[1] ARJAS, E. AND Norros, I. (1989) Change of life distribution via a hazard transformation: an inequality with application to minimal repair. Math. Operat. Res. 14, 355-361.

[2] Barlow, R. E. ANd Proschan, F. (1965) Mathematical Theory of Reliability. Wiley, New York.

[3] Bergman, B. (1985) On reliability theory and its applications. Scand. J. Statist. 12, 1-41.

[4] Finkelstein, M. S. (1991) Reliability of Technical Systems with Imperfect Repair (in Russian). Znanie, Moscow.

[5] NATVIG, B. (1990) On information-based minimal repair and the reduction in remaining system lifetime due to the failure of a specific module. J. Appl. Prob. 27, 365-375. 